\title{
Facteurs biologiques responsables des variations observées dans la précision des méthodes de routine utilisées pour le dosage de la matière grasse du lait
} (Milko-Tester, Infra-Red-Milk Analyser et Gerber)

\author{
par \\ R. GRAPPIN et R. JEUNET \\ (avec la collaboration technique de R. RIGOGNE) \\ I.N.R.A., Station Expérimentale Laitière (39) Poligny
}

Pour les dosages de routine de la matière grasse du lait, on dispose actuellement de trois méthodes dont les principes sont très différents :

- la méthode Gerber qui mesure le volume de la graisse extraite au moyen d'un acide,

- l'appareil Infra-Red-Milk Analyser qui mesure le nombre de liaisons ester des glycérides à la longueur d'onde de $5,73 \mu \mathrm{m}$,

- et l'appareil Milko-Tester qui repose sur une mesure turbidimétrique d'une émulsion de matière grasse du lait.

Dans un précédent travail consacré aux essais de l'appareil Milko-Tester (Grappin et Jeunet, 1970) nous avions souligné qu'en raison de leur principe, la précision de ces méthodes devait dépendre de certaines propriétés particulières de la graisse : masse volumique pour la méthode Gerber, coefficient d'extinction pour l'InfraRed-Milk Analyser et indice de réfraction pour le Milko-Tester ; ces différentes propriétés étant elles-mêmes le reflet de la composition de la matière grasse du lait, notamment la nature et les proportions relatives des différents acides gras. Or, on sait que la composition en acides gras varie en fonction de nombreux facteurs naturels comme la saison, l'alimentation, le stade de lactation, etc. Par conséquent, on peut s'attendre à ce que tout facteur qui fera varier de façon sensible la composition du lait en acides gras influence la précision des méthodes de dosage.

Compte tenu de l'importance de ce problème, il nous a paru utile de préciser les relations qui existent entre ces facteurs naturels de variation de la composition du lait et la précision des méthodes de dosage de la matière grasse. 
Notre étude a porté sur les trois méthodes de routine citées plus haut ; cependant, nous ne présenterons de façon détaillée que les résultats concernant le Milko-Tester car c'est le seul appareil nouveau actuellement utilisé en France. Les résultats concernant les deux autres méthodes seront donnés à titre de comparaison.

\section{MATERIEL ET METHODES}

\section{a) Choix des animaux}

Le schéma expérimental a été établi de façon à pouvoir contrôler et étudier séparément les facteurs suivants : saison, troupeau, individu et stade de lactation. L'étude a porté sur 6 troupeaux de la région de Poligny ayant chacun de 20 à 35 vaches de race Montbélairde, soit, au total, 145 vaches. Chaque mois, nous avons choisi parmi les 6 troupeaux, 42 animaux ( 7 par troupeau) de façon à ce qu'il y ait un nombre approximativement égal d'animaux en début, milieu et fin de lactation. De plus, de par le choix des 42 vaches sélectionnées chaque mois, nous avons pu suivre environ 5 vaches dans chaque troupeau pendant tout ou partie de leur lactation.

\section{b) Prélèvements}

L'étude a porté sur une année complète (de mars 1970 à février 1971). Tous les prélèvements ont été effectués une fois par mois (le 15 de chaque mois) sur le lait de la traite du soir. Immédiatement après les prélèvements, les échantillons étaient additionnés de 1 p. 1000 de $\mathrm{Cr}_{2} \mathrm{O}_{z} \mathrm{~K}_{2}$, répartis en flacons en fonction des différents dosages à effectuer et mis à $4^{\circ} \mathrm{C}$ jusqu'au moment du dosage.

\section{c) Analyses chimiques}

1) Dosage de la matière grasse

- Méthodes : Le taux de matière grasse des échantillons a été déterminé par les méthodes suivantes : Röse-Gottlieb (R. G.), (Norme FIL 1 : 1955), Gerber (Terroine, 1961), Milko-Tester MK III (M. T.), (Grappin et Jeunet, 1970) et Infra-Red-Milk Analyser (I.R.M.A.), (Adda et al., 1968). Les dosages I.R.M.A. n'ont porté que sur 11 mois en raison d'une défaillance du matériel. Toutes les déterminations ont été faites en double. Les résultats sont exprimés en gramme par kilo de lait.

- Etalonnage et contrôle de l'étalonnage des appareils MilkoTester et I.R.M.A. : Dans cet essai, le problème le plus important ne résidait pas dans l'étalonnage des appareils (cf. $\S$ d) mais dans le contrôle permanent de cet étalonnage. En effet, le but de cette étude étant de déceler l'influence (parfois très taible) des facteurs de variation de la composition de la matière grasse sur les résultats 
des dosages, il fallait s'assurer de la parfaite stabilité des appareils de façon à ne pas imputer à tel ou tel facteur, des variations qui seraient dues à une dérive ou à un mauvais fonctionnement des appareils. 4

Dans ce but, en dehors des systèmes de contrôle dont sont munis les appareils (valeur de shift pour le Milko-Tester et filtres étalons pour I.R.M.A.), nous avons mis en place un système de contrôle assez complexe qui comprenait, chaque mois et pour chaque appareil, le dosage :

$1^{\circ}$ de deux laits (un lait riche et un lait pauvre en matière grasse) reconstitués à partir d'un poids donné de poudre de lait gras provenant d'un lot unique utilisé pendant toute la durée de l'essai,

$2^{\circ}$ d'un lait de contrôle (lait de grand mélange) confectionné chaque mois et analysé une première fois en même temps que les échantillons du mois et gardé à $4^{\circ} \mathrm{C}$ pour être analysé une seconde fois en même temps que la série du mois suivant,

$3^{\text {o }}$ des échantillons de lait provenant de la série prélevée et déjà analysée le mois précédent.

Ces différents contrôles indépendants ont permis, le cas échéant, (notamment pour I.R.M.A.) de modifier les résultats de façon à ce qu'on puisse les considérer comme provenant d'appareils stables. De ce fait, la totalité des variations qui ont été constatées dans la précision des mesures peuvent être imputées directement aux facteurs étudiés.

\section{2) INDICE DE RÉFRACTION DE LA MATIÈRE GRASSE}

L'indice de réfraction $\left(n_{\mathrm{D}}\right)$ a été déterminé chaque mois sur les échantillons moyens de chaque troupeau ainsi que sur les laits individuels des vaches suivies pendant toute leur lactation. Les déterminations ont été faites avec un réfractomètre Zeiss Abbe en utilisant la méthode de Pont (1955) pour l'extraction de la graisse.

\section{d) Analyses statistiques}

Rappelons que la précision d'une méthode indirecte de dosage "y " - dans notre travail « y " sera la méthode Gerber, M.T. ou I.R.M.A. - est définie par la moyenne $(\bar{e})$ et l'écart-type $\left(\sigma_{\mathrm{e}}\right)$ des écarts algébriques entre les résultats donnés par la méthode y et les résultats de la méthode de référence " $\mathrm{x}$ » - ici la méthode RöseGottlieb - . Pour exprimer la dispersion des résultats, on utilise également, à la place de $\sigma_{\mathrm{e}}$, l'écart-type résiduel $\left(\sigma_{\mathrm{r}}\right)$, déduit de l'analyse de la variance de la régression de y sur $\mathrm{x}$. Les deux expressions $\sigma_{\mathrm{e}}$ et $\sigma_{\mathrm{r}}$ sont identiques (à un degré de liberté près) lorsque le coefficient de régression $b_{r}, x$ n'est pas différent de 1 ; dans le cas contraire, $\sigma_{\mathrm{e}}$ est supérieur à $\sigma_{\mathrm{r}}$. Rappelons qu'un appareil est correctement calibré si la droite de régression passe par l'origine et possède une pente égale à 1 . 
Comme dans le cas des appareils, il est possible de faire varier à volonté le calibrage et par conséquent la valeur de $b_{x}, x$ nous avons préféré utiliser l'écart-type résiduel $\sigma_{\mathrm{r}}$ plutôt que $\sigma_{\mathrm{e}}$ pour exprimer la précision des méthodes, car $\sigma_{r}$ dépend très peu de la façon dont ont été calibrés les appareils.

La plus grande partie de notre étude a donc porté sur l'analyse des régressions entre $\mathrm{x}$ et $\mathrm{y}$. De ce fait, comme nous l'avons signalé plus haut, l'exactitude de l'étalonnage initial des appareils n'était pas indispensable.

Dans la pratique, précisons que ce sont les valeurs de $\sigma_{\mathrm{e}}$ et de $\bar{e}$ qui sont importantes car elles représentent à la fois la valeur propre de la méthode et la façon dont est calibré et utilisé un appareil.

Soulignons enfin que la linéarité de la régression entre $\mathrm{y}$ et $\mathrm{x}$ n'est vraie, en particulier pour I.R.M.A. et le M.T. que dans des limites de taux de matière grasse comprises entre 20 et $70 \mathrm{~g} / \mathrm{kg}$ environ.

\section{RESULTATS}

\section{I. - ETUDE DU MILKO-TESTER}

\section{A) Influence de la saison, du troupeau et du stade de lactation}

Rappelons qu'en raison du schéma employé, les résultats donnés ci-dessous traduisent l'influence propre de chaque facteur étudié : la saison (qui comprend les différences d'alimentation, d'éclairement, de température, etc.), le troupeau et le stade de lactation, sans interaction des différents facteurs.

\section{1) RÉGRESSIONS ET MOYENNES}

- Suivant le critère de classement des données : saison (mois de calendrier), troupeau ou stade de lactation, l'analyse de covariance des résultats Milko-Tester (y) et Röse-Gottlieb (x) indique une influence très hautement significative $(\mathrm{P}<0,001)$ de la saison sur les résultats $\mathrm{M}$.T., une influence significative $(0,01<\mathrm{P}<0,05)$ du troupeau et non significative $(P>0,05)$ du stade de lactation.

D'autre part, sauf dans le cas du classement des données en fonction du stade de lactation, les tests de non parallélisme des droites de régression ont été trouvés significatifs, indiquant par là que la droite de régression (ou d'étalonnage) ne varie pas parallèlement à elle-même, en particulier dans le cas de la saison. De ce fait, l'interprétation des tests concernant les différences d'ordonnées à l'origine est en partie faussée, notamment dans le cas des facteurs " troupeau » et "stade de lactation " pour lesquels les tests F ont été trouvé pour le premier peu significatifs et non significatifs pour le second. De plus, pour l'étude de ces deux derniers facteurs, l'influence 

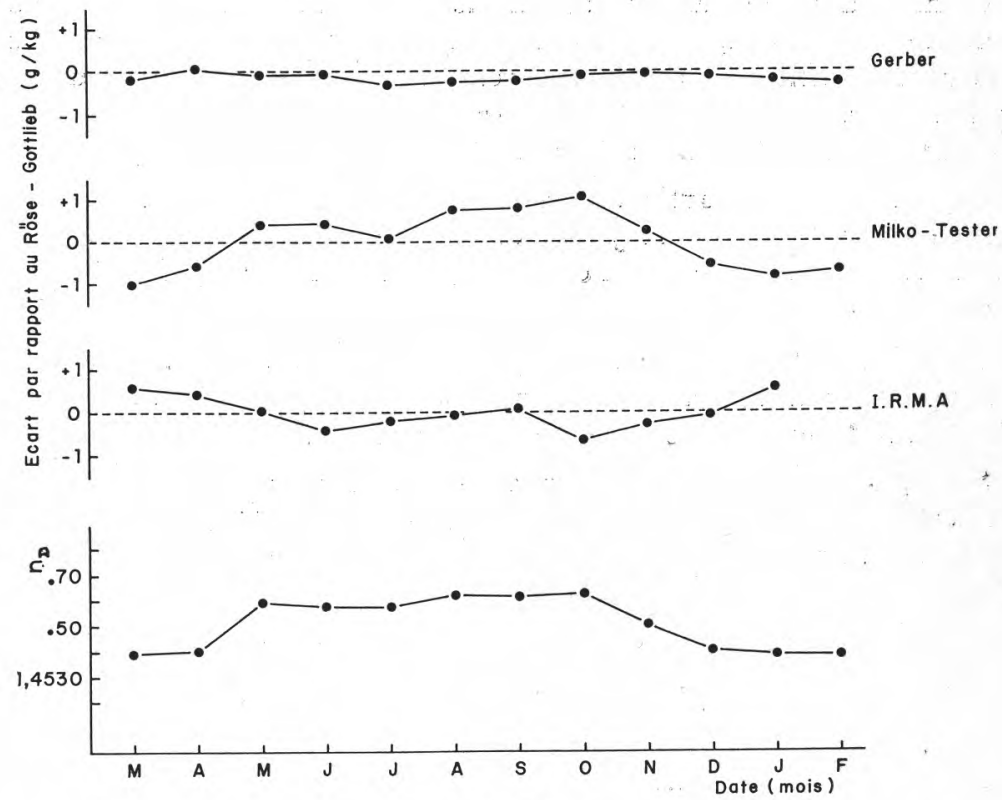

fig. 1

Influence de la saison sur la précision des méthodes Gerber, Milko-Tester et I.R.M.A. (écart par rapport à la méthode Röse-Gottlieb) et sur l'indice de réfraction $\left(\mathrm{n}_{\mathrm{D}}\right)$ de la matière grasse.

Moyennes mensuelles sur 42 laits individuels à tous les stades de lactation.
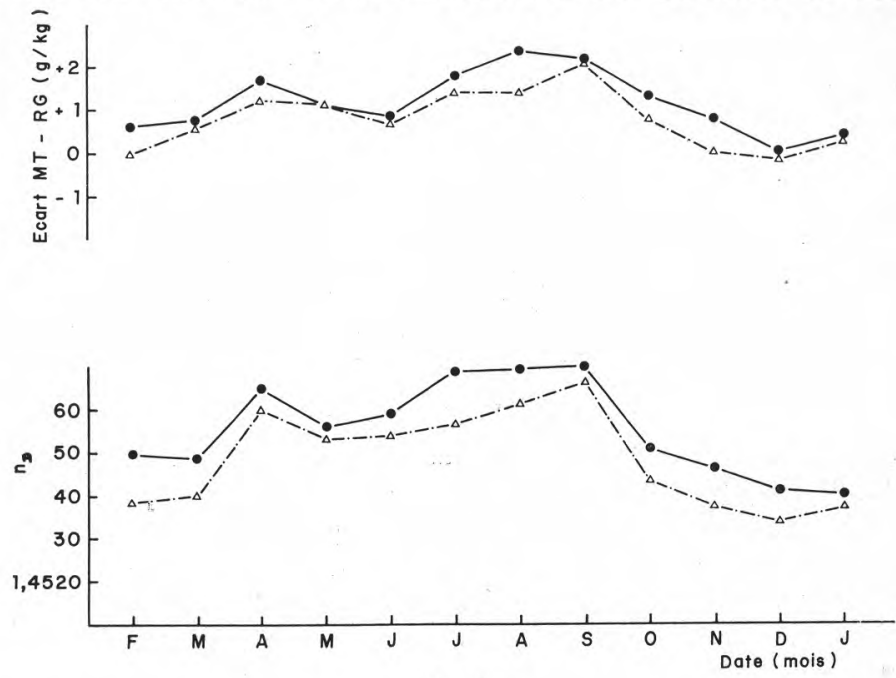

fig. 2

Influence du troupeau sur la précision du Milko-Tester (écart par rapport à la méthode Röse-Gottlieb) et sur l'indice de réfraction $\left(n_{D}\right)$ de la matière grasse. 


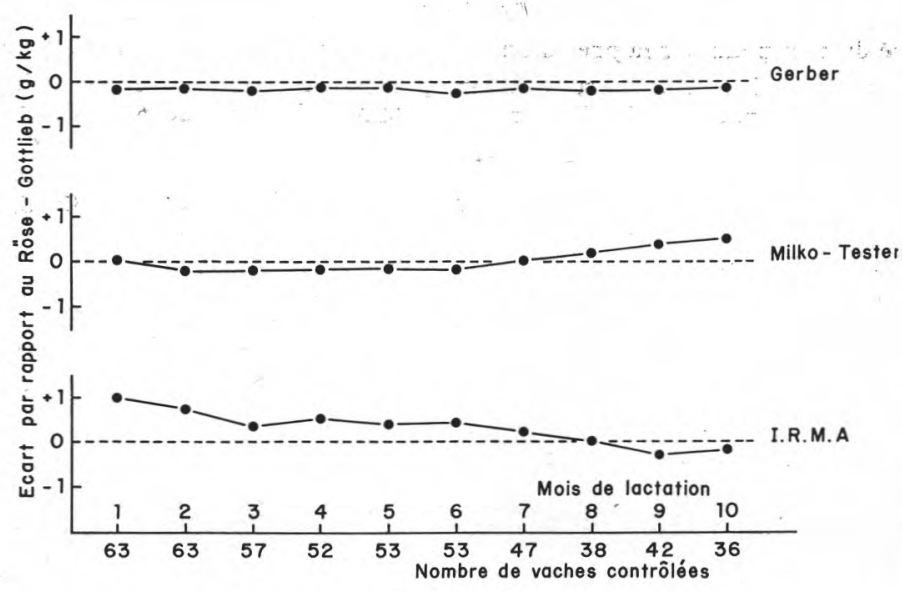

fig. 3

Influence du stade de lactation sur la précision des méthodes Gerber, Milko-Tester et I.R.M.A. (écart par rapport à la méthode Röse-Gottlieb).
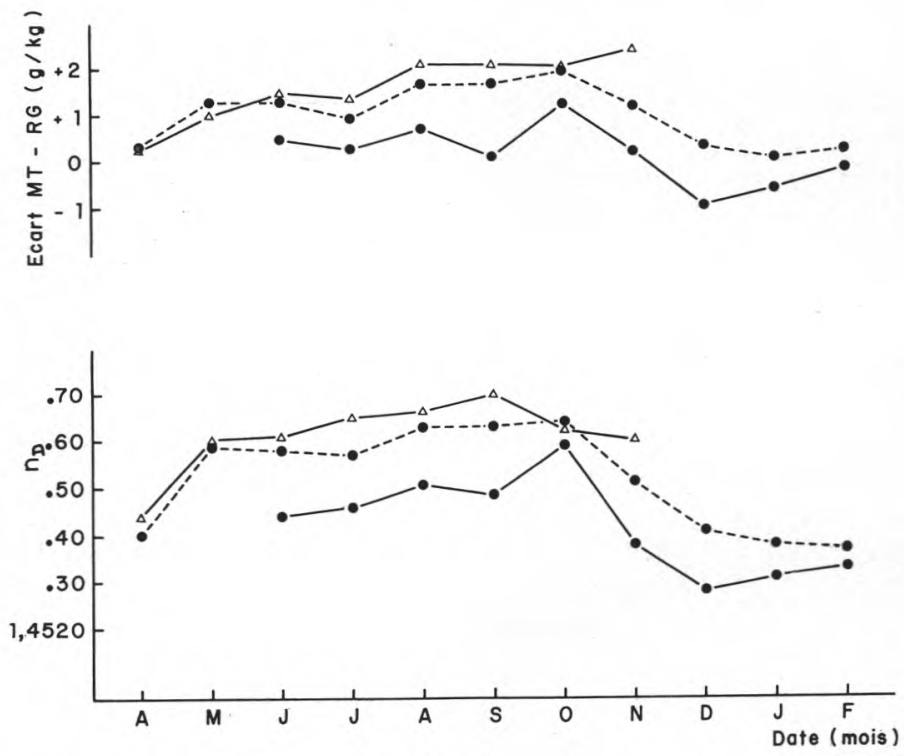

fig. 4

Influence de la vache sur la précision de la méthode Milko-Tester (écart par rapport à la méthode Röse-Gottlieb) et sur l'indice de réfraction $\left(\mathrm{n}_{\mathrm{D}}\right)$ de la matière grasse.

Comparaison des résultats obtenus sur 2 vaches au cours de leur lactation.

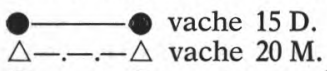

$\triangle \ldots .-\triangle$
vache $20 \mathrm{M}$. 


\section{TABLEAU 1}

Influence du troupeau sur la précision des méthodes de dosage de la matière grasse. Moyennes annuelles des écarts par rapport à la méthode Röse-Gottlieb des résultats Gerber, Milko-Tester et I.R.M.A. des laits de 6 troupeaux.

\begin{tabular}{c|c|c|c}
\hline \multirow{2}{*}{ Troupeau } & \multicolumn{2}{|c}{ Ecarts par rapport au Röse-Gottlieb $(\mathrm{g} / \mathrm{kg})$} \\
\cline { 2 - 3 } & Gerber & M.T. & I.R.M.A. \\
\cline { 2 - 3 } B & -0.18 & $-0,05$ & $-0,03$ \\
N & $-0,11$ & $-0,01$ & $-0,15$ \\
V & $-0,16$ & $+0,26$ & $-0,19$ \\
M & $-0,16$ & $-0,05$ & $-0,09$ \\
D & $-0,11$ & $-0,07$ & $+0,32$ \\
P & $-0,10$ & $-0,08$ & $+0,13$ \\
\hline
\end{tabular}

très marquée de la saison sur les résultats Milko-Tester a entraîné des variances résiduelles très élevées, masquant ainsi les effets factoriels.

- L'examen des moyennes obtenues avec le Milko-Tester et la méthode Röse-Gottlieb confirme en grande partie ces premiers résultats.

a) Saison : La figure 1 montre les variations saisonnières des écarts M.T.-R.G., dont l'amplitude est d'environ $2,0 \mathrm{~g} / \mathrm{kg}$ entre le mois de mars et le mois d'octobre. On note une différence moyenne très nette de $1,3 \mathrm{~g} / \mathrm{kg}$ environ, entre les périodes d'herbage (mai à novembre) et de stabulation (de décembre à avril), avec de brusques variations au moment des changements de régime.

b) Troupeau : Le tableau 1 donne pour les 6 étables la moyenne annuelle des écarts M.T.-R.G. Bien que l'amplitude des écarts entre les différents troupeaux soit assez faible $(0,34 \mathrm{~g} / \mathrm{kg})$, la figure 2 illustre clairement pour deux d'entre eux, leur influence sur la précision du Milko-Tester.

c) Le stade de lactation : Contrairement aux résultats de l'analyse de covariance, l'examen de la figure 3 semble indiquer une légère influence du mois de lactation sur les dosages Milko-Tester. Après une baisse sensible des écarts pendant le $1^{\text {er }}$ mois, on remarque une élévation lente mais régulière qui s'accentue en fin de lactation.

2) VARIANCES RÉSIDUELles

En classant les données suivant la saison, le troupeau ou le mois de lactation, on diminue la variance résiduelle $\left(\sigma_{r}^{2}\right)$ calculée pour 
l'ensemble des données, respectivement de 63 p. 100,4 p. 100 et 2 p. 100 ce qui traduit très nettement l'influence prépondérante de la saison sur la précision du Milko-Tester.

On obtient ainsi un écart-type de $0,50 \mathrm{~g} / \mathrm{kg}$ dans le cas d'un classement en fonction de la saison, contre $0,84 \mathrm{~g} / \mathrm{kg}$ pour l'ensemble des données non classées.

\section{B) Influence de l'individu}

Nous n'utiliserons ici que les résultats des vaches suivies jusqu'au $5^{\text {me }}$ mois au moins de leur lactation. Comme ces animaux ont vêlé entre les mois de mars et d'octobre, nous avons éliminé l'influence de la saison en corrigeant les résultats Milko-Tester en fonction des écarts moyens constatés au cours des différents mois.

L'analyse de la variance des écarts M.T.-R.G. montre une influence individuelle hautement significative à l'intérieur des étables (tab. 2). Sur 253 vaches ayant subi seulement 3 contrôles, Klijn et Posthumus (1970) trouvent également un effet significatif de l'animal.

Compte tenu de l'influence faible mais significative du troupeau (tabl. 2), les différences individuelles vont se trouver amplifiées si on considère une population d'individus appartenant à plusieurs troupeaux. Pour illustrer ces différences, nous avons choisi, parmi notre lot de 25 vaches, le cas extrême de 2 vaches, pour lesquelles la moyenne des écarts M.T.-R.G. est de $+1,6 \mathrm{~g} / \mathrm{kg}$ dans un cas (vache $20 \mathrm{M}$ ) et seulement de $+0,2 \mathrm{~g} / \mathrm{kg}$ dans l'autre cas (vache $15 \mathrm{D}$ ), soit, entre les 2 vaches, une différence de $1,4 \mathrm{~g} / \mathrm{kg}$ pour les résultats Milko-Tester (fig. 4).

\section{C) Relation avec l'indice de réfraction et la composition du lait en acides gras}

Pour tous les facteurs dont l'influence sur les résultats MilkoTester a été trouvée significative, nous pouvons vérifier sur les figures 1 (saison), 2 (troupeau) et 4 (individu), l'étroite corrélation entre les écarts M.T.-R.G. et l'indice de réfraction de la matière grasse, confirmant ainsi nos précédents travaux sur cette question (Jeunet et Grappin, 1970).

En ce qui concerne la saison, les variations d'indice de réfraction constatées au cours de nos essais sont en accord avec les nombreux travaux déjà consacrés à ce sujet. Par exemple, pour l'hémisphère Nord, Walstra (1965) note en Hollande une différence d'indice de réfraction de $15.10^{-4}$ entre les mois de mars et d'octobre. KuzdzalSavoie (1965), Guyot et Piraux (1965), Hutton et al. (1969), Mattson et al. (1969), Huyghebaert et Hendrickx (1970), constatent également des indices de réfraction (ou indice d'iode) plus élevés en été qu'en 
$T A B L E A U$ 2. - Influence du troupeau et de l'individu sur la précision du Milko-Tester

Analyse de la variance des écarts Milko-Tester - Röse-Gottlieb obtenus sur 25 vaches réparties dans 5 troupeaux et suivies pendant leur lactation

\begin{tabular}{l|c|c|c|c}
\hline \multicolumn{1}{c|}{ Origine } & $\begin{array}{c}\text { Somme des carrés } \\
\text { des écarts }\end{array}$ & D. L. & Carré moyen & F \\
\hline Troupeau & 2,8008 & 4 & 0,7002 & $3,43(+)$ \\
Individu (intra-troupeau) & 10,7194 & 20 & 0,5359 & $2,62(++)$ \\
Résiduelle (intra-individu) & 35,1038 & 172 & 0,2041 & \\
Totale & & 196 & & \\
\hline
\end{tabular}

$T A B L E A U$ 3. - Influence du troupeau sur la précision des dosages Milko-Tester

Analyse de la variance des écarts Milko-Tester - Röse-Gottlieb des laits de 15 troupeaux

\begin{tabular}{l|c|c|c|c|c}
\hline \multicolumn{1}{c|}{ Origine } & $\begin{array}{c}\text { Somme des carrés } \\
\text { des écarts }\end{array}$ & D.L. & $\begin{array}{c}\text { Carré } \\
\text { moyen }\end{array}$ & $\begin{array}{c}\text { Estimation de la } \\
\text { variance }\end{array}$ & F \\
\hline Entre troupeaux & 5,27 & 14 & 0,3764 & $\sigma_{\mathrm{e}}^{2}+10 \sigma^{2}$ & ++ \\
Intra-troupeaux (erreur) & 14,91 & 135 & 0,1104 & $\sigma_{\mathrm{e}}^{2}$ & \\
Totale & 20,18 & 149 & & \\
\hline
\end{tabular}


hiver et notent une bonne corrélation entre ces indices et la proportion d'acide oléique. D'une manière générale, la saison (ou plus exactement les différences de régime alimentaire entre les saisons) se traduit par une baisse de la proportion des acides gras à chaînes moyennes ou courtes (nombre d'atomes de carbone inférieur ou égal à 16) pendant les mois d'été et par une élévation de la proportion des acides gras longs en $\mathrm{C} 18$. En particulier, la proportion d'acide oléique (C $18: 1$ ) augmente, selon les auteurs et la nature des laits, de 5 à $10 \mathrm{p} .100,48 \mathrm{~h}$ après la mise à l'herbe. Signalons que l'allure des courbes de variation d'indice d'iode est différente suivant les régions (Kuzdzal-Savoie, 1956 ; Jamotte et Piraux, 1957).

Nous avons vu que la précision du Milko-Tester ne variait guère avec le stade de lactation. Nous ne disposons pas, à ce sujet de résultat concernant les variations d'indice de réfraction, cependant d'après Decaen et Adda (1970) il y a une chute brutale et importante de la proportion d'acides gras longs au début de la lactation, suivie d'une légère augmentation. Kuzdzal-Savoie (1956) rapporte également une baisse de l'indice d'iode au début de la lactation suivie d'une augmentation à partir du deuxième mois. Si nos résultats concernant les différences M.T.-R.G. ne traduisent pas aussi nettement ces modifications de composition, ils ne sont cependant pas en contradiction avec les conclusions de ces auteurs.

Au niveau individuel, l'analyse de la variance des indices de réfraction (corrigés en fonction de la saison) obtenus pour les vaches suivies pendant tout ou partie de leur lactation, montre une influence hautement significative $(\mathrm{P}<0,01)$ de l'individu, rejoignant ainsi les conclusions de l'analyse des écarts M.T.-R.G. faite au paragraphe précédent. Ces résultats sont illustrés par la figure 5 qui donne, pour 28 vaches, la relation entre la moyenne par lactation des indices de réfraction et la moyenne des écarts M.T.-R.G.

L'indice de réfraction apparaît donc nettement comme étant une caractéristique individuelle. Nous avons d'ailleurs pu confirmer cette hypothèse en vérifiant sur un petit nombre de vaches au cours de 2 lactations successives, la bonne répétabilité de la valeur de l'indice de réfraction de la matière grasse.

\section{COMPARAISONS AVEC LA METHODE GERBER ET L'APPAREIL I.R.M.A.}

\section{a) Gerber}

L'examen des résultats montre que la méthode Gerber n'est pratiquement pas influencée par les quatre facteurs étudiés. 
Suivant le mode de classement des données, les amplitudes des différences moyennes Gerber-R.G. sont les suivantes : $0,39 \mathrm{~g} / \mathrm{kg}$ pour la saison (fig. 1), 0,19 g/kg pour le stade de lactation (fig. 3) et $0,08 \mathrm{~g} / \mathrm{kg}$ entre les 6 troupeaux (tab. 1) ; quant aux variations ind1viduelles, l'écart-type des moyennes par lactation est faible : $0,11 \mathrm{~g} / \mathrm{kg}$ (fig. 6).

Ces résultats laissent penser que la masse volumique de la matière grasse, qui est le facteur principal en cause dans cette méthode, n'est pas modifiée de façon sensible par les différents facteurs étudiés.

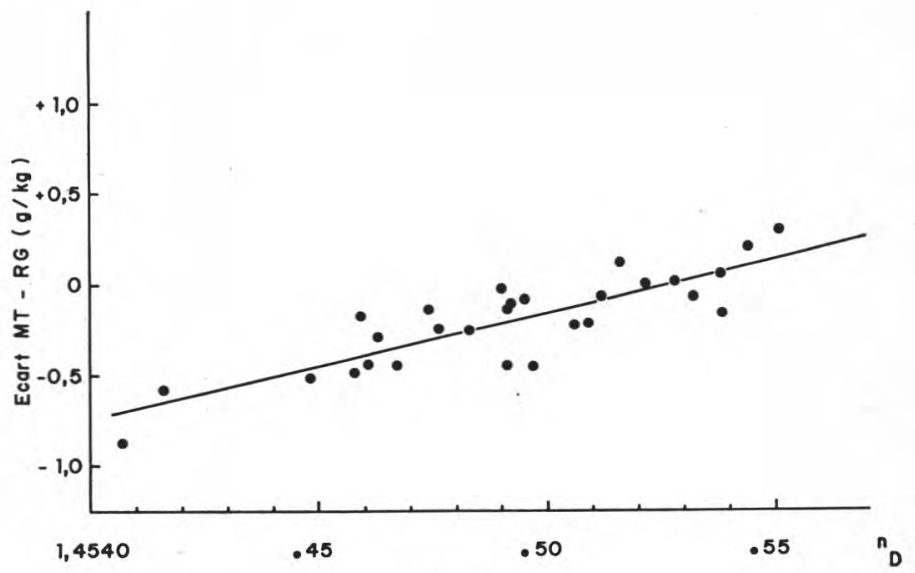

fig. 5

Relation entre les moyennes par lactation des écarts Milko-Tester Röse-Gottlieb et les indices de réfraction $\left(\mathrm{n}_{\mathrm{D}}\right)$ de la matière grasse. $\mathrm{n}=28$ vaches; $\mathrm{r}=0,86 ; \mathrm{b}=0,059$
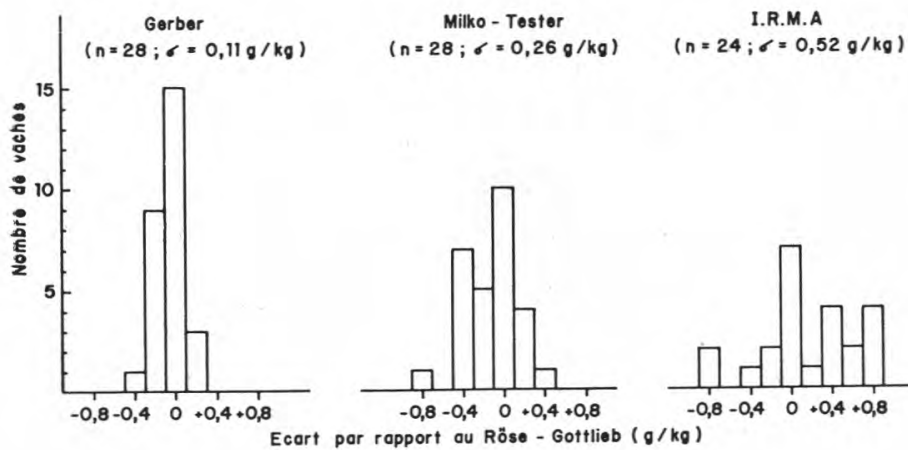

Ecart par rapport ou Röse - Gottlieb $(\mathrm{g} / \mathrm{kg})$

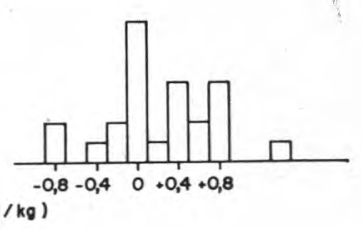

fig. 6

Influence de la vache sur la précision des méthodes Gerber, MilkoTester et I.R.M.A. Histogrammes des moyennes par lactation, des écarts par rapport à la méthode Röse-Gottlieb 


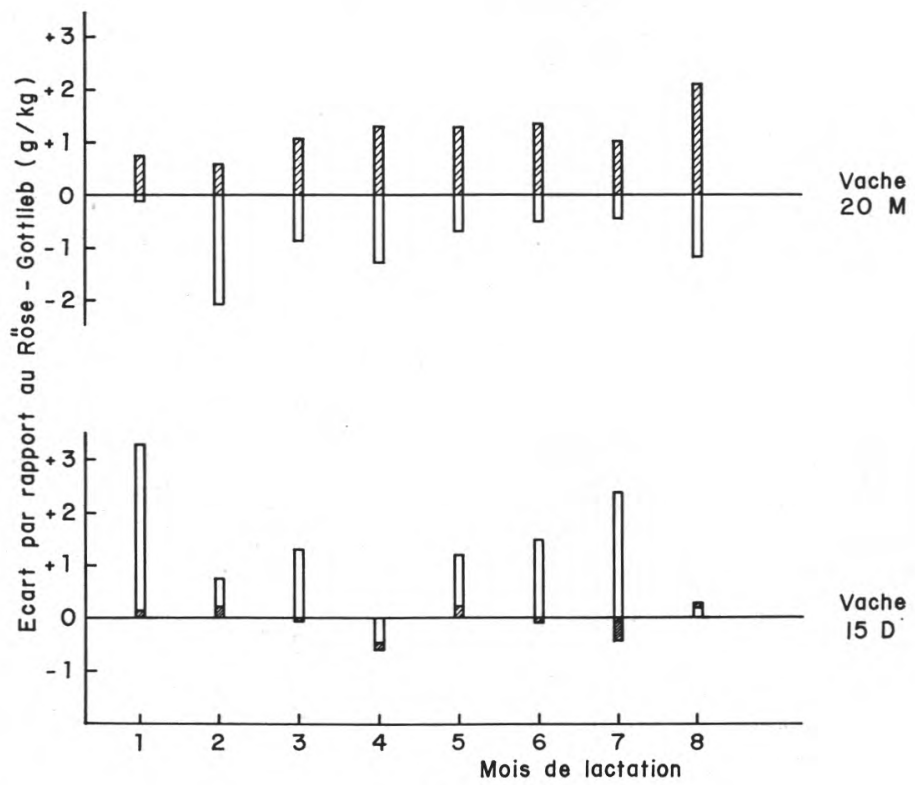

fig. 7

Comparaison des résultats Milko-Tester (hachuré) et I.R.M.A. (blanc) obtenus sur deux vaches $(20 \mathrm{M}$ et $15 \mathrm{D})$ pendant leur lactation (résultats corrigés en fonction de la saison)

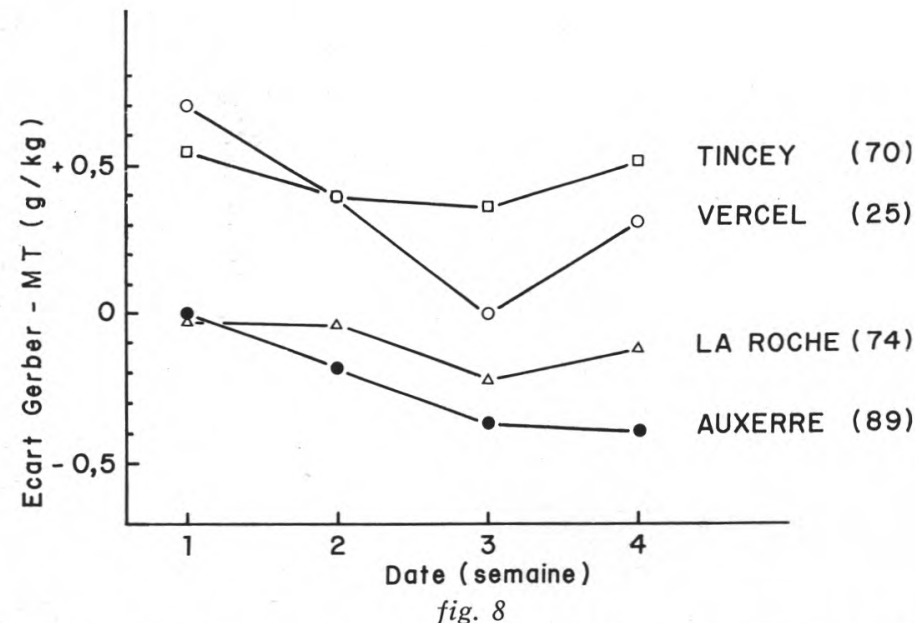

Influence de la "région » sur les écarts entre le Milko-Tester et la méthode Gerber

Comparaison des résultats obtenus pendant 4 semaines consécutives sur 4 régions de l'Est de la France 


\section{b) I.R.M.A.}

L'appareil I.R.M.A. est sensible aux mêmes facteurs de variation que le Milko-Tester, avec des variations d'amplitude à peu près semblables mais de sens opposé.

- Pour les variations saisonnières (fig. 1, on note une amplitude des écarts I.R.M.A.-R.G. 1,5 g/ $\mathrm{kg}$ environ entre les mois d'octobre et de janvier et une différence assez nette entre les mois d'été et d'hiver. Mais, à l'inverse du Milko-Tester, l'I.R.M.A. donne des résultats par défaut pendant les mois d'été.

- Le stade de lactation paraît avoir une influence plus marquée sur les résultats I.R.M.A. que sur les résultats Milko-Tester (fig. 3). On constate en effet une baisse régulière assez importante des écarts I.R.M.A.-R.G. avec le mois de lactation.

- L'influence de l'individu sur les résultats I.R.M.A. est très nette : sur 24 vaches contrôlées pendant toute leur lactation, l'écarttype des moyennes par lactation des écarts I.R.M.A.-R.G. s'élève à $0,52 \mathrm{~g} / \mathrm{kg}$ (fig. 6). Comme pour les autres facteurs étudiés, cette influence s'exerce en sens opposé de celui du Milko-Tester. A titre d'exemple, nous donnons dans la figure 7 les écarts M.T.-R.G. et I.R.M.A.R.G. (corrigés en fonction de la saison) qui ont été observés sur deux animaux pendant leur lactation. Avec la vache $20 \mathrm{M}$ les résultats Milko-Tester sont, en moyenne, constamment supérieurs au RöseGottlieb $(+1,21 \mathrm{~g} / \mathrm{kg})$ et les résultats I.R.M.A. constamment inférieurs $(-0,9 \mathrm{~g} / \mathrm{kg})$; par contre, la situation inverse se produit avec la vache $15 \mathrm{D}$, pour laquelle on obtient une moyenne des écarts nulle pour le Milko-Tester et égale à $+1,3 \mathrm{~g} / \mathrm{kg}$ pour I.R.M.A.

On peut expliquer ces résultats de la façon suivante : l'augmentation de la proportion des acides gras longs et en particulier des acides insaturés, provoque une augmentation de l'indice de réfraction de la matière grasse qui, nous le savons, entraîne une surestimation des résultats Milko-Tester. Mais, en même temps, il y a une diminution de la proportion des acides gras à moyenne et courte chaîne et par conséquent une diminution du nombre de liaisons ester par unité de poids. Cette modification se répercute, cette fois par une sous-estimation des lectures I.R.M.A.

En fait, tout facteur, comme l'alimentation par exemple dont nous reparlerons plus loin, ou la saison, qui tend à modifier la proportion des acides gras en $\mathrm{C}_{1 \mathrm{~s}}$ aura des répercussions inverses sur les deux méthodes.

\section{DISCUSSION}

Pour tous les facteurs étudiés, nos résultats confirment l'hypothèse couramment admise selon laquelle la précision de la méthode Gerber est à peu près indépendante des variations de la composition du lait en acides gras. 
Le problème se limite donc, pour l'instant, aux méthodes I.R.M.A. et Milko-Tester.

1) Dans cette étude, nous n'avons abordé qu'un nombre limité de facteurs. Pour connaître l'influence des autres facteurs importants, comme la région, la race, l'alimentation ou la traite, d'autres expérimentations seront nécessaires. Cependant, on peut, dès à présent, apporter quelques éléments de réponse. En effet, nous avons montré qu'il existe une assez bonne corrélation entre, d'une part la précision des méthodes et d'autre part certaines propriétés physiques de la graisse du lait qui sont elles-mêmes en étroite relation avec la composition du lait en acides gras. Comme on dispose, pour ces différents facteurs, de plusieurs travaux concernant leur influence sur la composition des acides gras, une brève revue de ces travaux constitue une bonne approche du problème.

- La région: Au même titre que la saison, la région recouvre plusieurs facteurs. Les principaux sont la race, le climat et le mode d'alimentation. Bien qu'il n'y ait pas d'enquête précise sur les différences régionales de composition de la matière grasse du lait en acides gras, il est à peu près certain que ces différences existent. Ainsi, dans une enquête portant sur quatre régions françaises, Kuzdzal-Savoie (1956) enregistre entre ces régions, des différences assez marquées dans les variations saisonnières de l'indice d'iode. En Suède, Mattson et al. (1969) et en Belgique, Jamotte et Piraux (1957) constatent des différences régionales, plus faibles cependant que les variations saisonnières. Hall (1970) observe également que l'amplitude des variations saisonnières de la composition en acides gras diffère suivant les régions. Pour notre part, nous avons constaté, sur une période d'un mois, des différences hautement significatives des écarts M.T.-Gerber entre les laits de quatre départements de l'Est de la France (fig. 8).

- Race : Hutton et al. (1969) trouvent des modifications assez importantes de composition en acides gras entre deux lots de vaches de race Frisonne et Ayrshire. Ils notent, en particulier, une élévation deux fois plus importante des taux d'acide oléique au moment de la mise à l'herbe pour les Frisonnes. Ils attribuent une partie de cette différence à des dates de vêlages variables selon les deux lots.

Kuzdzal-Savoie (1959) constate des différences d'indice d'iode entre les races Parthenaise et Normande mais cette différence est environ quatre fois plus faible que les variations saisonnières. En revanche, dans un troupeau expérimental contrôlé pendant 4 semaines consécutives, nous n'avons trouvé aucune différence significative entre deux lots identiques de 15 vaches Holstein et 15 vaches Normande (Grappin et Colleau, 1971).

- Alimentation : $\mathrm{Si}$, comme nous l'avons signalé plus haut, les variations de composition en acides gras entre le régime de pâturage et celui de stabulation sont assez bien définies, en revanche, l'influence exacte des différents régimes alimentaires qui sont pratiqués 
en France pendant les périodes de stabulation ou de pâturage, est plus délicate à établir.

On sait cependant qu'une consommation importante de tourteaux modifie l'indice d'iode de la graisse : par exemple, le tourteau de coprah abaisse l'indice d'iode, celui du soja ou du lin l'augmente (Kuzdzal-Savoie, 1965).

Certains ensilages (Kuzdzal-Savoie, 1961), de même que l'espèce fourragère (Decaen et Ghadaki, 1970) ont également une influence.

D'une manière générale, on peut considérer que l'influence de l'alimentation sur la nature et la proportion des différents acides gras du lait est liée au processus de synthèse de ces acides par la mamelle : les acides gras en C 18 ont pour origine les graisses alimentaires et les réserves corporelles, tandis que les acides gras ayant 14 atomes de carbone et moins, proviennent des produits de fermentation du rumen ; quant à l'acide palmitique $(C 16)$ il a une origine double (Decaen et Journet, 1967 ; Jones, 1969). Ainsi, avec un régime pauvre en lipide, Delage et Fehr (1967) constatent une baisse du taux butyreux du lait accompagné d'une diminution de la proportion des acides gras en C 18. Mais ils soulignent que l'amplitude des variations de composition de la matière grasse du lait est nettement plus faible que celle du régime alimentaire. Kuzdzal-Savoie (1965) note également l'importance des facteurs physiologiques de régulation qui diminuent notablement l'influence de l'alimentation.

- Traite : La traite semble avoir une influence faible mais très significative sur les propriétés de la matière grasse. Kuzdzal-Savoie et Kuzdzal (1961) indiquent que les laits de la traite du soir ont un indice d'iode constamment supérieur aux laits de la traite du matin. Mc Dowell (1970) confirme ce résultat en mesurant les indices de réfraction.

2) De l'ensemble de cette étude expérimentale et bibliographique on peut dégager quatre facteurs importants : la saison, la région, le troupeau et l'individu. Une question importante se pose : est-il possible par des adaptations convenables des méthodes et en particulier grâce à des réglages appropriés des appareils, de réduire ou mieux d'annuler l'influence de ces facteurs de façon à améliorer la précision des résultats ?

- En ce qui concerne la saison, il est possible, pour une région donnée, d'éliminer son influence en vérifiant régulièrement les résultats par rapport à une méthode de référence et en procédant à une modification de l'étalonnage des appareils. Cependant, dans le cas particulier d'un laboratoire analysant le lait de plusieurs régions pour lesquelles les dates de mise à l'herbe et de rentrée à l'étable sont très différentes, il y aura, pendant l'intervalle de temps qui sépare les dates de changement de régime dans chaque région, des écarts systématiques entre les résultats des dosages de matière grasse du lait de ces régions. 
- Les différences entre régions, troupeaux ou individus sont plus délicates. En effet, en raison d'une part de leur caractère systématique et d'autre part du fait qu'elles n'affectent pas de la même manière la totalité des échantillons de lait analysés, ces différences seront, à l'inverse des différences saisonnières, très difficiles à éliminer.

Pour supprimer les grandes différences régionales, chaque laboratoire devra, en pratique, procéder à l'étalonnage de ses propres appareils. Par contre, si à l'intérieur de la zone d'activité d'un laboratoire il y a plusieurs « régions naturelles » très différentes, le travail du laboratoire s'en trouvera compliqué car il faudra faire, pour chaque région, un étalonnage particulier.

Quant aux différences entre troupeaux ou entre individus, il est bien évident qu'elles ne peuvent pas être annulées. Dans ce cas le problème est de savoir si ces différences sont compatibles avec les objectifs de paiement et de sélection. En d'autres termes, quelle erreur commet-on dans l'estimation du taux mensuel des laits de troupeaux (cas du paiement) ou du taux moyen par lactation (cas du contrôle laitier individuel), lorsqu'on utilise les appareils MilkoTester ou I.R.M.A. ?

Prenons à titre d'exemple, le cas des dosages avec le MilkoTester. On peut décomposer la variance totale des écarts M.T.-R.G. en une variance due au facteur contrôlé, $\sigma_{A}^{2}$ (troupeau ou individu) et une variance de l'erreur, $\sigma^{2}$ e. Si on opère $n$ contrôles pour estimer les moyennes (mensuelles ou par lactation) du facteur contrôlé, la variabilité de ces moyennes est mesurée par l'écart-type $\sigma=\sqrt{\sigma_{\mathrm{A}}^{2}+\frac{\sigma^{2}}{\mathrm{n}} \text {. }}$

- Pour estimer la variabilité des écarts M.T.-R.G. des laits de troupeaux, nous avons réalisé l'essai suivant : quinze troupeaux ont été contrôlés tous les 3 jours en matière grasse à l'aide du Milko-Tester et par la méthode Röse-Gottlieb, pendant une période de 1 mois. L'analyse de la variance des écarts M.T.R.G. confirme l'influence significative du troupeau (tab, 3). Les variances

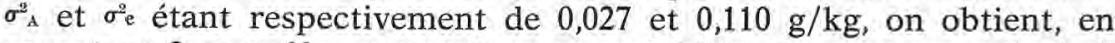
comptant 3 contrôles par mois, une variabilité des moyennes égale à $0,25 \mathrm{~g} / \mathrm{kg}$. Autrement dit, avec 3 prélèvements par mois, l'erreur sur la détermination du taux butyreux est inférieure à $\pm 0,5 \mathrm{~g} / \mathrm{kg}$ dans 95 p. 100 des cas. Sans être négligeable, elles est cependant environ 4 fois plus faible que l'erreur qui provient de la variabilité journalière du taux butyreux (Grappin et al., 1968).

- Pour l'estimation de la variabilité par lactation des écarts M.T.-R.G. des laits individuels, nous pouvons reprendre l'analyse de la variance donnée dans le tableau 2, mais cette fois, sans tenir compte de l'effet étable. On obtient alors respectivement des variances entre vaches $\left(\sigma_{\mathrm{A}}^{2}\right)$ et intra-vaches $\left(\sigma_{\mathrm{e}}^{2}\right)$ égales à 0,046 à $0,204 \mathrm{~g} / \mathrm{kg}$. Soit, avec 9 contrôles par lactation, une variabilité égale à $0,26 \mathrm{~g} / \mathrm{kg}$. L'erreur sur l'estimation du taux butyreux moyen par lactation est inférieur à $\pm 0,52 \mathrm{~g} / \mathrm{kg}$ dans $95 \mathrm{p} .100$ des cas. Ce dernier résultat 
est illustré par la figure 6 dans laquelle nous donnons pour le MilkoTester et pour les méthodes Gerber et I.R.M.A., la variabilité des moyennes par lactation des écarts par rapport au Röse-Gottlieb. On note des différences assez sensibles entre les trois méthodes avec, en particulier, un écart-type assez élevé de $0,52 \mathrm{~g} / \mathrm{kg}$ pour I.R.M.A.

\section{CONCLUSION}

L'utilisation de plus en plus courante, d'appareils automatiques basés sur des méthodes physiques pour estimer la composition chimique du lait, pose de nouveaux problèmes aux laboratoires spécialisés et, d'une manière générale, à tous ceux qui ont à juger et à utiliser ces méthodes.

Un premier problème concerne la précision des méthodes. Jusqu'à présent, les auteurs s'accordent pour donner à la précision d'une méthode des valeurs différentes, suivant qu'elle est appliquée à des laits individuels ou à des laits de troupeaux. Or, nous venons de montrer que la précision n'est pas seulement liée à l'origine du lait (individu ou troupeau) mais également à d'autres facteurs dont les principaux sont la saison et la "région ", celle-ci recouvrant un ensemble de facteurs secondaires (race, climat, alimentation, etc.). Autrement dit, donner une valeur à la précision d'une méthode n'a de sens que si l'on spécifie bien, pour tous les facteurs importants, la nature de l'échantillon qui a été utilisé pour estimer cette précision. En fait, la simple notion de précision ne suffit plus pour apprécier la valeur d'une méthode, il faut également que l'utilisateur connaisse les "facteurs biologiques » qui influencent cette précision. C'est la condition sine qua non pour obtenir des résultats satisfaisants. Et pour cela, il faut que l'utilisateur dispose d'une norme précise lui permettant de vérifier et régler en toutes circonstances l'étalonnage de son appareil. Autrement, il sera difficile d'obtenir des résultats homogènes, à l'intérieur et entre les différents laboratoires.

Un second problème résulte de la comparaison des résultats obtenus par plusieurs méthodes de dosage. Il présente deux aspects différents. Le premier intéresse les transformateurs, c'est celui des bilans " entrée " et "sortie " de matière grasse. Lorsque l'usine achète aux producteurs de lait une quantité de matière grasse mesurée à l'aide d'un appareil (I.R.M.A. par exemple) et vend, sous la forme de produits transformés, cette même quantité de matière grasse, mais estimée cette fois, à l'aide d'une méthode de référence, peut-elle équilibrer son bilan de matière grasse ? Nous savons que si l'appareil est correctement étalonné à partir de la méthode de référence, il ne doit pas y avoir de différences analytiques importantes. En effet, dans ce cas, les résultats donnés par les deux 
méthodes de mesures ont des moyennes identiques, elles diffèrent seulement au niveau des individus qui composent cette moyenne.

Le deuxième aspect concerne les deux appareils I.R.M.A. et Milko-Tester. Nous avons vu qu'il existe, entre les résultats donnés par ces deux appareils, des différences très sensibles qui tiennent aux principes mêmes des méthodes sur lesquelles ils reposent. Par conséquent, si dans un cadre donné, les deux appareils sont utilisés simultanément, il faudra utiliser et interpréter les résultats avec prudence.

Si la connaissance des "facteurs biologiques " de variation de la précision des méthodes de routine est indispensable sur le plan pratique, elle demeure cependant insuffisante. En effet, rappelons que ces facteurs agissent sur des "facteurs biochimiques » et que ce sont ces derniers qui en définitive influencent directement les méthodes de dosages. Grâce aux travaux déjà effectués dans ce domaine, nous avons pu en fournir quelques exemples dans notre travail. Cependant une connaissance plus approfondie de la nature de ces facteurs biochimiques et de leur mode d'action serait très utile pour apprécier la valeur des diverses méthodes étudiées.

\section{Rés u m é}

Dans un essai portant sur un an, nous avons déterminé l'influence de la saison, du stade de lactation, du troupeau et de l'individu, sur la précision des principales méthodes de routine de dosage de la matière grasse du lait : Gerber, Milko-Tester (M.T.) et Infra-Red-Milk Analyser (I.R.M.A.).

L'analyse statistique des différences entre les résultats donnés par chacune de ces méthodes et la méthode Röse-Gottlieb a montré que pour les appareils M.T. et I.R.M.A., l'influence est hautement significative pour la saison et l'individu, significative pour le troupeau et non significative pour le stade de lactation. La méthode Gerber n'est pas significativement influencée par les facteurs étudiés. Nous avons confirmé l'existence d'une étroite relation entre les variations d'indice de réfraction de la matière grasse (et par conséquent la composition du lait en acides gras) et la précision du M.T.

Nous avons constaté que l'influence des différents facteurs envisagés, sur les écarts M.T.-R.G. et I.R.M.A.-R.G., étaient de sens contraire.

Il apparaît, après une étude bibliographique complémentaire, que les facteurs les plus importants sont : la saison, la région, le troupeau et l'individu.

Les moyens pour atténuer ou annuler l'influence de certains facteurs, ainsi que les conséquences de l'emploi des appareils M.T. ou I.R.M.A. au niveau du paiement du lait et du contrôle laitier individuel, sont discutés. 


\section{S u m m a r y}

In order to estimate the influence of season, stage of lactation, herd and cow on the accuracy of the routine methods for milk fat determination (Gerber, Milko-Tester (M.T.), Infra-Red-Milk Analyser (I.R.M.A.), an experiment was carried out during one year.

The statiscal analysis of the differences between each of these methods and the Röse-Gottlieb method, has shown a highly significant influence for season and cow, a significant one for herd and a non significant one for stage of lactation, on M.T. and I.R.M.A. results The Gerber method is not significantly influenced by any of the se factors. The close relationship between the variation of the refractive index of fat (and consequently with the fatty acid composition of milk) and the accuracy of the Milko-Tester has been confirmed.

We have found opposite effects of the different factors studied on M.T. and I.R.M.A. readings.

Taking also into account previous results from various authors on this same subject it appears that the most important factors affecting the accuracy of M.T. and I.R.M.A. are : season, region, herd and cow.

The means for reducing or removing the influence of some of these factors and the consequences which are involved when using either M.T. or I.R.M.A. for milk payment or individual milk recording are discussed.

Reçu pour publication en décembre 1971.

\section{NOTE COMPLEMENTAIRE}

réalisée avec la collaboration de C. DECAEN, Mme LENTO et M. FOUILLEUX (1)

Afin de définir les relations existant entre la composition du lait en acides gras et la précision des appareils Milko-Tester et I.R.M.A., nous avons effectué les dosages des principaux acides gras (2) sur les échantillons de lait provenant des deux vaches $20 \mathrm{M}$ et $15 \mathrm{D}$ étudiées précédemment.

(1) Laboratoire de la Chaire de Zootechnie de l'E.N.S.A. de Rennes (35).

(2) Dosages effectués par chromatographie en phase gazeuse d'esters méthyliques (colonne D.E.G.S., $2,5 \mathrm{~m}, 180^{\circ} \mathrm{C}$ ) après extraction des matières grasses du lait par la méthode de Folch. Résultats exprimés en proportions pondérales d'esters méthyliques. 
Compte tenu du principe de chaque appareil et des résultats de notre expérimentation, il est apparu que la meilleure façon de relier la précision des appareils à la composition du lait en acide gras était de comparer :

$1^{\circ}$ la proportion des acides gras insaturés aux différences relatives M.T. - Röse-Gottlieb (fig. 9),

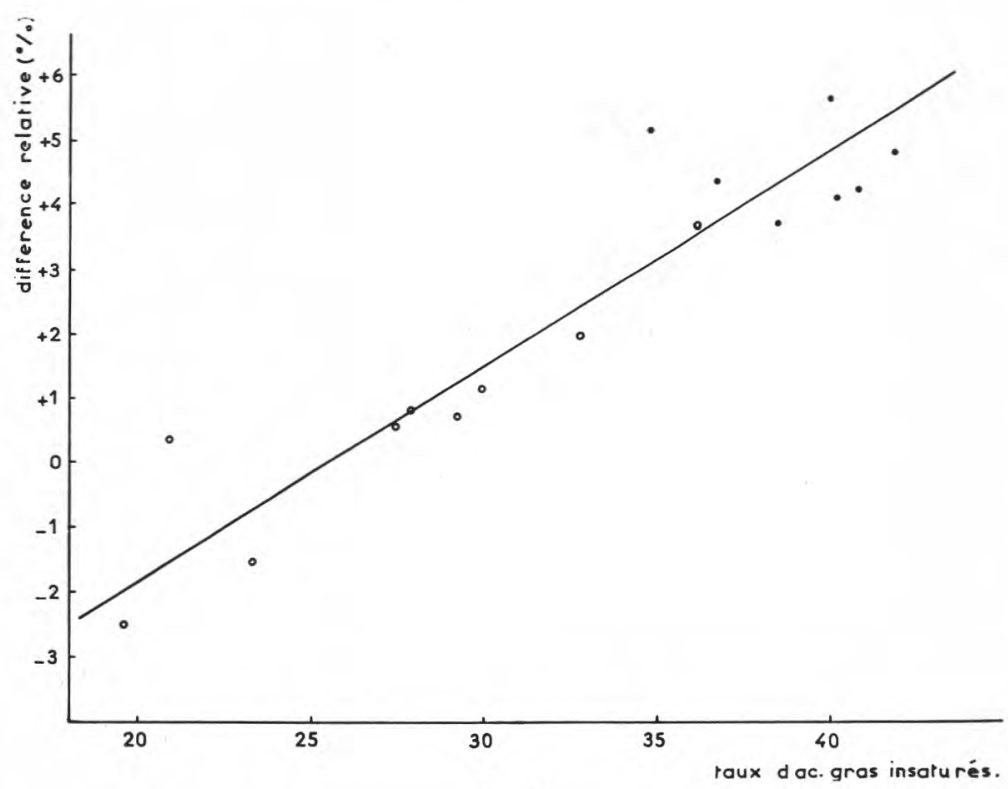

fig. 9

Relation entre la proportion des acides gras du lait et les différences relatives entre les résultats des dosages de matière grasse par l'appareil Milko-Tester et la méthode Röse-Gottlieb.

yache $15 \mathrm{D}$.

- vache $20 \mathrm{M}$.

$2^{\circ}$ le poids moléculaire moyen des acides gras aux différences relatives I.R.M.A. - Röse-Gottlieb (fig. 10) ; le poids moléculaire moyen étant directement lié à la longueur des chaînes d'acides gras et par conséquent au nombre de liaisons ester par unité de poids.

Pour chacune des comparaisons, nous obtenons des corrélations hautement significatives de $+0,95$ dans le cas du Milko-Tester et de — 0,71 pour l'appareil I.R.M.A.

La proportion des acides gras insaturés du lait, qui est liée à l'indice de réfraction de la graisse (pour notre échantillon $r=0,96$ ), 
explique la quasi-totalité des écarts observés entre le Milko-Tester et la méthode Röse-Gottlieb.

En revanche, la composition en acides gras, exprimée sous la forme du poids moléculaire moyen ne donne que partiellement (environ 50 p. 100 de la variance totale) l'origine des différences I.R.M.A. - Röse-Gottlieb.

Par ailleurs, si on relie d'une part la corrélation élevée $(r=0,89)$ qui existe entre le taux d'acides gras insaturés et le poids moléculaire moyen des acides gras, et d'autre part les corrélations exprimées plus haut et illustrées par les figures 9 et 10 , on explique aisé-

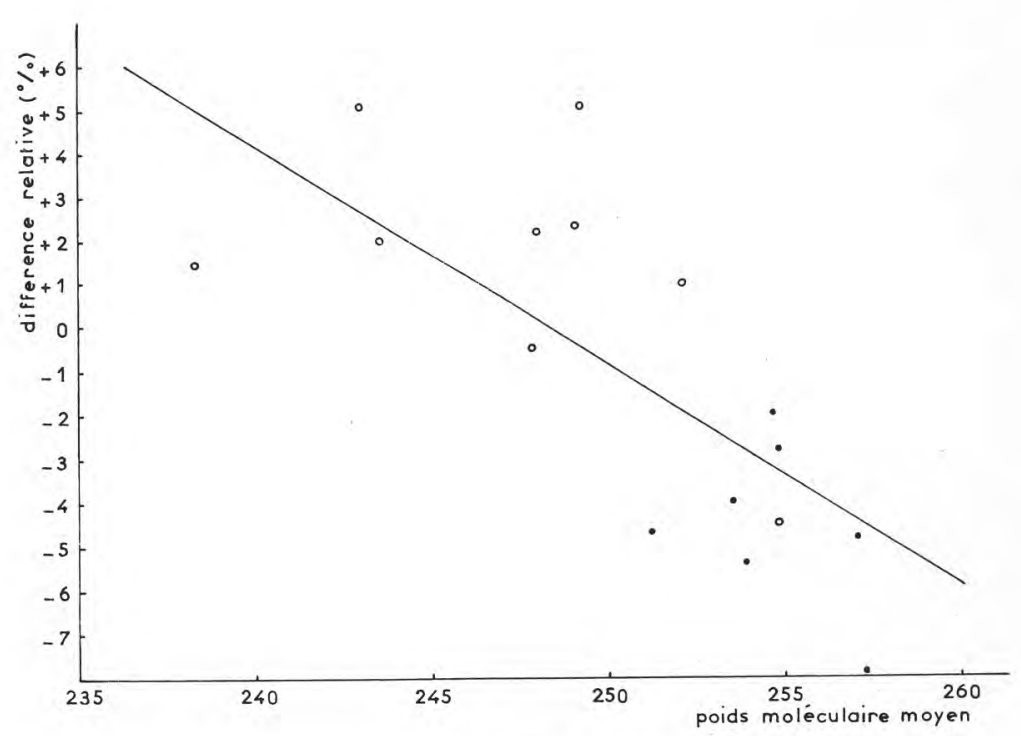

fig. 10

Relation entre le poids moléculaire moyen des acides gras du lait et les différences relatives entre les résultats des dosages de matière grasse par l'appareil I.R.M.A. et la méthode Röse-Gottlieb.

vache $15 \mathrm{D}$.

- vache $20 \mathrm{M}$.

ment que les deux méthodes Milko-Tester et I.R.M.A. « réagissent » avec une amplitude à peu près identique, mais en sens inverse, aux facteurs de variation de la composition du lait en acides gras.

Ces résultats expérimentaux sont en parfait accord avec les informations personnelles fournies par Walstra (1969) et confirment nos précédentes hypothèses. 


\section{Remerciements}

Nous remercions vivement le docteur Goulden du National Institute for Research in Dairying pour l'aide qu'il nous a apportée lors de la réalisation de ce travail.

Notre reconnaissance va également à tous ceux qui ont lu le manuscrit, Mme Kuzdzal, MM. Mocquot, Ricordeau et Adda. Leurs suggestions et critiques nous ont été précieuses.

Nous remercions également la Société Foss-electric pour le prêt d'un appareil Milko-Tester M K III.

\section{Références bibliographiques}

Adda (J.), Blanc-Patin (E.), Jeunet (R.), Grappin (R.), Mocouot (G.), Poujardieu (B.) et RicoRdEAU (G.) (1968). - Essais d'utilisation de l'Infra-Red-Milk Analyser. Le Lait, 48, 145-53, 293-302.

Decaen (C.) et Journet (M.) (1967). - Evolution au début de la lactation, de la sécrétion des principaux acides gras du lait et de la concentration en acides gras libres du sang chez la vache. Ann. Biol. Anim. Bioch. Biophys., 7, 137-144.

Decaen (C.) et AdDA (J.) (1970). - Evolution de la sécrétion des acides gras des matieres grasses au cours de la lactation de la vache. Ann. Biol. Anim. Bioch. Biophys., 10, 659-677.

DeCAeN (C.) et GHADAKI (M. G.) (1970). - Variation de la sécrétion des acides gras des matières grasses du lait de vache à la mise à l'herbe et au cours des six premières semaines d'exploitation du fourrage vert. Ann. Zootechn., 19, 393-412.

Delage (J.) et Fehr (P. M.) (1967). - Influence des lipides alimentaires sur la sécrétion des acides gras par la mamelle de chèvre. Ann. Biol. Anim. Bioch. Biophys., 7, 437-444, 445-457.

Mc Dowell (A. K. R.) (1970). - Diurnal variations in the composition of milk fat. XVIII Int. Dairy Congr., 1E : 601, in Dairy Sci. Abstr., 1970, 4790.

Grappin (R.) et Jeunet (R.) (1970), - Essais de l'appareil « Milko-Tester Automatique " destiné au dosage en série de la matière grasse du lait. Le Lait, 50, 235-256.

Grappin (R.) et Colleau (J. J.) (1971). - Résultats non publiés.

Guyot (A. L.) et Piraux (E. F.) (1965). - Etude par la chromatographie en phase gazeuse de la matière grasse du lait de vaches. Variations de la composition en acides gras observées en Belgique. Le Lait, 45, 603-620.

Hall (A. J.) (1970). - Seasonal and regional variations in the fatty acid composition of milk fat. Dairy Inds., 35, 20-24, in Dairy Sci. Abstr., 1970, 1773.

Hutton (K.), Seeley (R. C.) and ARmstong (R. G.) (1969). - The variation throughout a year in the fatty acid composition of milk fat from two dairy heards. J. Dairy Res., 36, 103-113.

Huyghebaert (A.) and Hendrickx (H.) (1970). - The relation between the fatty acid composition and the iodine value and refractive index of butterfat. Milchwissenschaft, 25, 506-510.

Jamotte (P.) et PirnuX (E.) (1957). - Indices de réfraction et caractéristiques de consistance des beurres belges. Bull. Inst. Agron. et Stat. Rech., Gembloux, $25,297-328$.

Jeunet (R.) et GRAPpin (R.) (1970). - Note sur la relation entre l'indice de réfraction de la matière grasse du lait et la précision des dosages de matière grasse par l'appareil Milko-Tester. Le Lait, 50, 654-657. 
JoNEs (E. A.) (1969). - Reviews of the progress of dairy science. Recent developments in the biochemistry of the mammary gland. J. Dairy Sci., 36, 145-167.

KLIJN (C. J.) and Posthumus (G.) (1970). - The determination of the fat content of milk for milk recording purposes with the Milko-Tester Automatique. Communication $17^{m e}$. Session du Comité Européen de Contrôle Laitier Beurrier, Salzbourg.

Kuzdzal-Savore (S.) (1956), - Les variations de composition de la matière grasse du beurre en fonction de la saison et du stade de lactation. $C . R X I V^{\text {me }}$ Congrès Int. Laits et Dérivés, Rome, vol. II.

Kuzdzal-Savoie (S.) (1959). - Influence de la race des vaches laitières sur la composition de la matière grasse du lait et la qualité du beurre. Ann. Techn. Agric., 8, 35-53.

Kuzdzal-Savoie (S.) et Kuzdzal (W.) (1961). - Les acides polyinsaturés du beurre. Influence de la saison.Ann. Techn. Agric., 10, 73-80.

Kuzdzal-Savoie (S.) (1961). - L'ensilage et la qualité des produits laitiers. Bull. Techn. Inf., $\mathrm{n}^{\circ}$ 164, 947-956.

Kuzdzal-Savoie (S.) (1965). - Influence de la composition de la ration alimentaire sur la composition chimique du beurre de vache. Ann. Techn. Agric., 14, $187-262$.

Mattsson (S.), Swartling (P.) and Nilson (R.) (1969). - The major fatty acids in whole milk fat and in a fraction obtained by crystallization from acetone. J. Dairy Res., 36, 169-175.

Pont (E. G.) (1955). - A de-emulsification technique for use the peroxyde test on the fat of milk cream, concentrated and dried milks. Aust., J. Dairy Techn., $\mathrm{n}^{\circ}$ mai-juin, 72.

Terroine (E.F.) (1951). - Dosage de la matière grasse du lait. Ann. Nutr. Alim., 5, 6.

Walstra (P.) (1965). - Over de brekingsindex van melkvet. Neth. Milk and Dairy J., 19, 1-7.

WALSTRA (P.) (1963). - Communication personnelle. 